\title{
Alignment and detection efficiency of CMS Preshower detector
}

\author{
Yeng-Ming Tzeng' \\ Department of Physics, National Taiwan University \\ No. 1, Sec. 4, Roosevelt Road, Taipei, 10617 Taiwan(R.O.C) \\ E-mail: ymtzeng@cern.ch \\ On behalf of the CMS Collaboration
}

\begin{abstract}
The Preshower detector, as part of the CMS Endcap electromagnetic calorimeter (ECAL), is designed to have good spatial resolution to measure the position of incoming particles and thus aid particle identification in the endcaps. It comprises two layers of lead absorbers, each followed by silicon strip sensors with $1.9 \mathrm{~mm}$ pitch. The physics performance of the Preshower relies upon excellent detection efficiency and accurate alignment to the Tracker and the Endcap ECAL crystals. Charged tracks from $7 \mathrm{TeV}$ collisions, reconstructed by the Tracker and extrapolated through the Preshower to the Endcap crystals, are used for this purpose. More than $99.8 \%$ of Preshower strips are operational, with detection efficiency better than $99.5 \%$. The alignment is measured with an accuracy of better than $1 \mathrm{~mm}$, meeting the specifications.
\end{abstract}

35th International Conference of High Energy Physics (ICHEP2010)

Paris,France

July 22-28, 2010

$1 \quad$ Speaker 


\section{Introduction}

The Preshower detector, as part of the CMS Endcap electromagnetic calorimeter (ECAL), is designed to have good spatial resolution to measure the position of incoming particles and thus aid particle identification in the endcaps [1]. In each endcap there are two orthogonal layers of $1.9 \mathrm{~mm}$-pitch silicon sensors, each preceded by thin layers of lead that initiate electromagnetic showers. The silicon layers are known as "ES+F, ES+R, ES-F, ES-R", where the nomenclature reflects the endcap (+ or -) and whether the layer is "Front" or "Rear". The physics performance of the Preshower relies upon excellent detection efficiency and accurate alignment to the Tracker and the Endcap ECAL crystals.

\section{Alignment with respect to Tracker}

As the tracker and endcap disks (supporting the EE and ES) are independent objects, there is a non-negligible probability of mis-alignment between them. High-purity charged tracks are used in this alignment study for both endcaps. The tracks are extrapolated to the ES to predict the location of expected hits. Only the central region of ES $(1.7<\eta<2.3)$ is used for this study. Matching hits are found using the closest reconstructed hit on ES within a search window. A $\chi^{2}$ is defined as a sum of squared residuals following a normal distribution. Thus, 3 alignment parameters are obtained by minimizing the $\chi^{2}$ iteratively for all selected tracks in all events. Figure 1 shows the critical parts of residuals for each ES plane, both before and after software alignment for ES+.
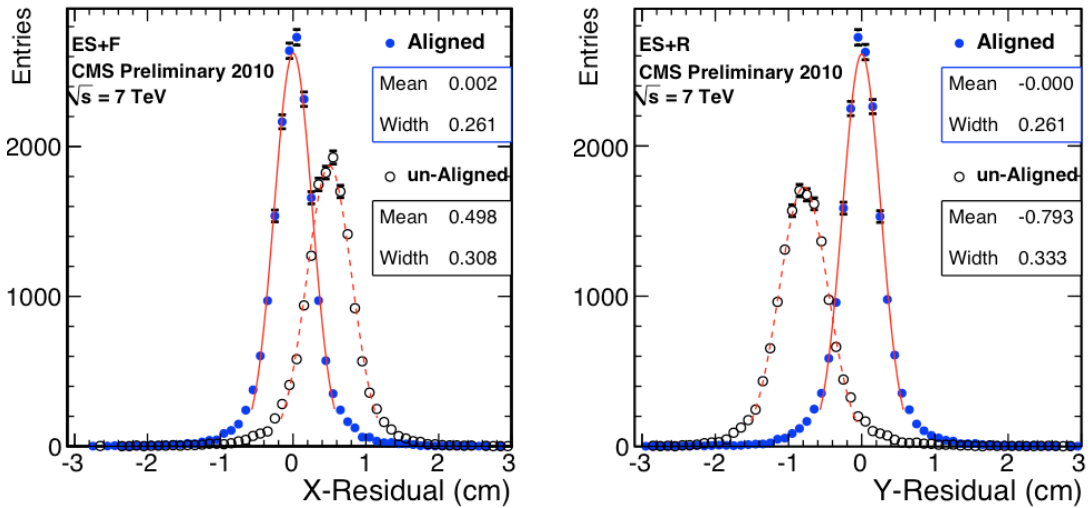

Figure 1 : The left and right plots show the alignment with respect to the tracker in the $\mathrm{X}$ (horizontal, where the "F" plane gives the most accuracy) and $\mathrm{Y}$ (vertical, using the "R" plane) directions respectively.

\section{Alignment with respect to $\mathrm{EE}$}

The ES disks are supported by a conical structure attached to the backplate of the endcap hadron calorimeter. The procedure for aligning the ES to the EE is similar to the alignment of the ES to the Tracker. Charged particles, without isolation or e/ $\gamma$ identification requirements, producing EE energy clusters with $\mathrm{E}_{\mathrm{T}}>2 \mathrm{GeV}$ are used as the source of this study. Figure 2 shows the alignment between the EE and ES in the positive endcap for data and simulation, 
showing an excellent agreement. As the EE and ES are permanently mechanically aligned by the same support structures there is less opportunity for mis-alignment. Indeed the "out of the box" mis-alignment between ES and EE is less than half a millimeter in all planes.

\section{Tracker-ES hit-matching efficiency}

The hit-matching efficiency is defined as the probability of finding a hit in the ES within a window around a predicted hit, given by extrapolations of charged tracks from the primary vertex. The efficiency is actually a convolution of the efficiencies of the tracking, the extrapolation to the ES and the ES itself. Figure 3 shows this efficiency, averaged over all four ES planes, as a function of track $\mathrm{p}_{\mathrm{T}}$ for $7 \mathrm{TeV}$ minimum bias collision data and simulation $(\mathrm{MC})$. The $\mathrm{p}_{\mathrm{T}}$ dependence is due to imperfection of the track reconstruction algorithm, mainly fake tracks. The ES hit efficiency for $\mathrm{p}_{\mathrm{T}}>7 \mathrm{GeV}$ is, in $\mathrm{MC}, 100 \%$. The agreement between data and $\mathrm{MC}$ demonstrates that the plateau below $100 \%$ is indeed due to inefficiencies in the track extrapolation.
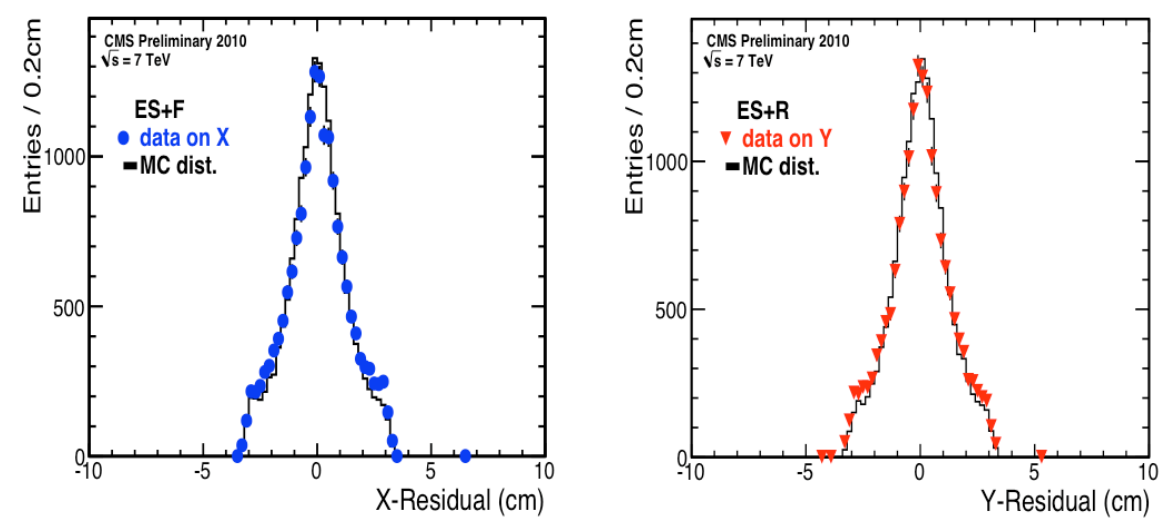

Figure 2 : The left and right plots show the alignment of ES+ with respect to EE+ in $\mathrm{X}$ and $\mathrm{Y}$ directions respectively.

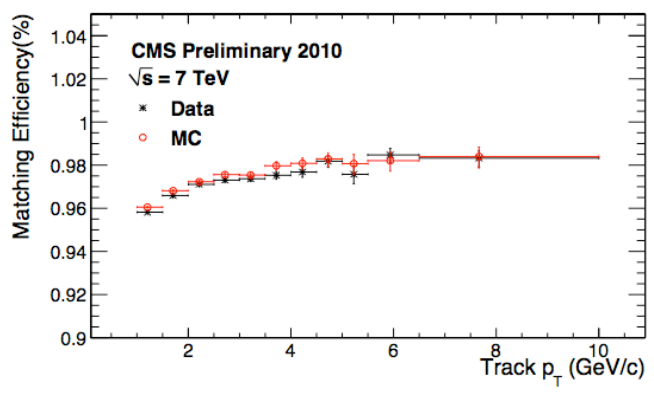

Figure 3 : The hit-matching efficiency of the preshower agrees well with expectation.

\section{Summary}

A relative mis-alignment in the vertical positions of the Tracker with respect to both ES endcaps is about $8 \mathrm{~mm}$. A horizontal mis-alignment between the Tracker and the positive ES is about $5 \mathrm{~mm}$, whilst for the other endcap it is $0 \mathrm{~mm}$. The mis-alignment between the EE and ES is less than half a millimeter in all planes. The ES hit-matching efficiency in $7 \mathrm{TeV}$ data is over 
$98 \%$, consistent with that expected from MC [2] and indicates a true hit efficiency of better than $99.5 \%$ given by a comparison of data and MC.

\section{References}

[1] "The CMS Experiment at the CERN LHC", 2008 JINST 3 S08004

[2] "Electromagnetic calorimeter commissioning and first results with $7 \mathrm{TeV}$ data", CMS NOTE-2010/012 